\title{
A Polynomial-time Algorithm for a Stable Matching Problem with Linear Valuations and Bounded Side Payments
}

\author{
Rashid FAROOQ \\ Research Institute for Mathematical Sciences, \\ Kyoto University, Kyoto 606-8502, Japan. \\ Email: farooq@kurims.kyoto-u.ac.jp
}

\begin{abstract}
We study an extension of the Gale-Shapley marriage model and the ShapleyShubik assignment model by considering linear valuations and bounded side payments. Our model includes the Eriksson-Karlander hybrid model as a special case. We propose a polynomial-time algorithm which finds a pairwise-stable outcome.
\end{abstract}

Keywords: stable marriage model, assignment game

\section{Introduction}

A two-sided matching market consists of two disjoint finite sets of agents. The purpose is to match the agents of opposite sides in pairs. A matching is a set of pairs of opposite sides such that each agent appears at most once and is called stable if there is no pair of agents who are not matched with each other but prefer each other to their partners in the matching.

The marriage model by Gale and Shapley [4] and the assignment model by Shapley and Shubik [10] are well known in the theory of two-sided markets. In [4], Gale and Shapley proposed an algorithm that always finds a (perfect) stable matching for any instance of a stable marriage model problem. In their model, side payments are not allowed, i.e., the agents are rigid. In the assignment model by Shapley and Shubik [10], in contrast to the Gale-Shapley marriage model, side payments are permitted, i.e., the agents are flexible, and they showed that the core is non-empty.

Kaneko [5] unified both the Gale-Shapley marriage model and the Shapley-Shubik assignment model, and proved the non-emptiness of the core, but does not consider lattice property. Roth and Sotomayor [9] proposed a general model that includes the both marriage and assignment model. They showed the existence of stable outcome and investigated the lattice property for payoffs in the core. The model of Eriksson and 
Karlander [1] deals with both rigid and flexible agents, which is a common generalization of the marriage and assignment models. The existence of a stable outcome and the lattice property of the set of stable outcomes are preserved in their model. Following the idea of Eriksson and Karlander [1], Sotomayor [11] investigated their hybrid model and gave a non-constructive proof of the existence of a pairwise-stable outcome.

Very recently, Fujishige and Tamura [2] proposed a common generalization of the marriage model and the assignment model by utilizing the framework of discrete convex analysis which was developed by Murota $[6,7,8]$. Their model also includes hybrid models of Eriksson and Karlander [1] and Sotomayor [11]. The existence of a pairwisestable outcome is preserved in their model. They further extended their model in [3] by assuming possibly bounded side payments and proved the existence of a pairwise-stable outcome. In their work, however, structure of the set of pairwise-stable outcome is not discussed.

In the present work, our aim is to formulate a model which includes the Gale-Shapley marriage model, the Shapley-Shubik assignment model and the Eriksson-Karlander model as special cases. We use the notion of bounded side payments and valuations rather than rigidity and flexibility. We list here the main assumptions in our model:

- the set of agents is partitioned into two sets; the set of men and the set of women,

- each agent has at most one partner of opposite side,

- side payments are permitted,

- side payments are bounded by lower and upper bounds,

- valuations of agents for the side payments from opposite side are identified by linear and strictly increasing real valued functions.

We can handle rigid agents by assuming $\mathbf{0}$ as a lower and upper bound for the side payments and the flexible agents can be dealt by considering lower and upper bounds of the side payments sufficiently small and sufficiently large, respectively, and hence the marriage model, the assignment model and the Eriksson-Karlander hybrid model are included in our model. We propose a polynomial-time algorithm in the number of agents to find a pairwise-stable matching.

This paper is organized as follows: In Section 2, we discuss our model and give a comparison between known models and our model. In Section 3, we characterize the pairwise stability. We will use this characterization to develop a polynomial-time algorithm. Section 4 deals with the case when valuations are linear. In this section, first of all, we give several lemmas which will help us to design our algorithm. We then propose our algorithm and finally discuss its correctness and complexity. 


\section{Model Description}

Let $M$ and $W$ be two disjoint finite sets of agents and let $E=M \times W$, i.e., the set of all pairs $(i, j)$ of agents $i \in M$ and $j \in W$. A subset $X \subseteq E$ is called a matching if every agent appears at most once in $X$. Given a matching $X, k \in M \cup W$ is called unmatched in $X$ if it does not appear in $X$; otherwise matched in $X$.

Before describing our model, we briefly explain the marriage model by Gale and Shapley [4] and the assignment model by Shapley and Shubik [10]. In the marriage model, $M$ and $W$ represent the sets of men and women, respectively. Each man has preferences on the women and each woman has preferences on the men. Negotiations and side payments are not involved in this model. We represent the preferences of men and women by the numbers $a_{i j} \in \mathbf{R}$ and $b_{i j} \in \mathbf{R}$, respectively, for all $(i, j) \in E$. For $i \in M$ and $j_{1}, j_{2} \in W$, if $a_{i j_{1}}>a_{i j_{2}}$ then we say that $i$ prefers $j_{1}$ to $j_{2}$, and $i$ is indifferent between $j_{1}$ and $j_{2}$ if $a_{i j_{1}}=a_{i j_{2}}$. Similarly, the preferences of women over men are defined by the vector $\left\{b_{i j} \mid(i, j) \in E\right\}$. Here we assume that $a_{i j}>0$ if $j$ is acceptable to $i$, and $a_{i j}=-\mu$ otherwise, and $b_{i j}>0$ if $i$ is acceptable to $j$, and $b_{i j}=-\mu$ otherwise, where $\mu>0$ is a sufficiently large number. A matching $X$ is called pairwise-stable if there exist $q \in \mathbf{R}^{M}$ and $r \in \mathbf{R}^{W}$ such that

(m1) $q_{i}=a_{i j}$ and $r_{j}=b_{i j}$ for all $(i, j) \in X$,

(m2) $q \geq \mathbf{0}, r \geq \mathbf{0}$, and $q_{i}=0$ (resp. $r_{j}=0$ ) if $i$ (resp. $j$ ) is unmatched in $X$,

(m3) $q_{i} \geq a_{i j}$ or $r_{j} \geq b_{i j}$ for all $(i, j) \in E$.

Gale and Shapley [4] presented an algorithm which finds a stable matching in this model.

In the assignment game, $M$ and $W$ represent the sets of sellers and buyers, respectively. The negotiation and side payments between agents of both sides are allowed. Naturally, each agent wants to gain as much profit as possible from his/her partner. Here $a_{i j} \in \mathbf{R}$ and $b_{i j} \in \mathbf{R}$ represent the profits of $i$ and $j$, respectively, when $i$ and $j$ are matched. The preferences of sellers over buyers and of buyers over sellers can be defined similarly as in the marriage model. A matching $X$ is called pairwise-stable if there exist $q \in \mathbf{R}^{M}$ and $r \in \mathbf{R}^{W}$ such that

(a1) $q_{i}+r_{j}=a_{i j}+b_{i j}$ for all $(i, j) \in X$,

(a2) $q \geq \mathbf{0}, r \geq \mathbf{0}$, and $q_{i}=0$ (resp. $\left.r_{j}=0\right)$ if $i$ (resp. $j$ ) is unmatched in $X$,

(a3) $q_{i}+r_{j} \geq a_{i j}+b_{i j}$ for all $(i, j) \in E$.

Shapley and Shubik [10] showed the existence of a stable outcome in this assignment model.

Now we describe our model. For each $(i, j) \in E$ we denote by $\nu_{i j}: \mathbf{R} \rightarrow \mathbf{R}$ a valuation of agent $i \in M$ for a side payment from $j \in W$ to $i$, and by $\nu_{j i}: \mathbf{R} \rightarrow \mathbf{R}$ 
a valuation of agent $j \in W$ for a side payment from $i \in M$ to $j$. We assume that $\nu_{i j}$ and $\nu_{j i}$ are continuous and monotone increasing and that there exist the inverse functions $\nu_{i j}^{-1}$ and $\nu_{j i}^{-1}$ over $\mathbf{R}$ for all $(i, j) \in E$. We also assume that we are given vectors $l, u \in \mathbf{R}^{E}$ with $l \leq u$, where $l_{i j}$ and $u_{i j}((i, j) \in E)$ denote the lower and upper bounds of a side payment from $j \in W$ to $i \in M$.

We say that $p=\left(p_{i j}:(i, j) \in E\right) \in \mathbf{R}^{E}$ is a feasible side payment vector from $W$ to $M$ if $l_{i j} \leq p_{i j} \leq u_{i j}$ for all $(i, j) \in E$. A pair $(X, p)$ of a matching $X$ and a feasible side payment vector $p$ is said to be a pairwise-stable outcome if $q \in \mathbf{R}^{M}$ and $r \in \mathbf{R}^{W}$ defined by

$$
\begin{aligned}
& q_{i}=\left\{\begin{array}{cl}
\nu_{i j}\left(p_{i j}\right) & \text { if } i \text { is matched with } j \text { in } X \\
0 & \text { if } i \text { is unmatched in } X
\end{array} \quad(\forall i \in M),\right. \\
& r_{j}=\left\{\begin{array}{cl}
\nu_{j i}\left(-p_{i j}\right) & \text { if } j \text { is matched with } i \text { in } X \\
0 & \text { if } j \text { is unmatched in } X
\end{array} \quad(\forall j \in W)\right.
\end{aligned}
$$

satisfy

(S1) $q \geq \mathbf{0}$ and $r \geq \mathbf{0}$,

(S2) $q_{i} \geq \nu_{i j}(c)$ or $r_{j} \geq \nu_{j i}(-c)$ for each pair $(i, j) \in E$ and each $c \in\left[l_{i j}, u_{i j}\right]$.

For $i \in M$ and $j_{1}, j_{2} \in W, \nu_{i j_{1}}\left(p_{i j_{1}}\right)>\nu_{i j_{2}}\left(p_{i j_{2}}\right)$ means $i$ prefers $j_{1}$ to $j_{2}$ and $i$ is indifferent between $j_{1}$ and $j_{2}$ if $\nu_{i j_{1}}\left(p_{i j_{1}}\right)=\nu_{i j_{2}}\left(p_{i j_{2}}\right)$ for a feasible side payment vector $p$. Here $q_{i}$ and $r_{j}$ represent the payoffs for $i \in M$ and $j \in W$, respectively. If there exist a pair $(i, j) \in E$ and $c \in\left[l_{i j}, u_{i j}\right]$ such that $q_{i}<\nu_{i j}(c)$ and $r_{j}<\nu_{j i}(-c)$, then $i$ and $j$ had better change their current status or partners and make a partnership with each other.

By defining the linear valuations as

$$
\nu_{i j}\left(p_{i j}\right)=a_{i j}+p_{i j}, \quad \nu_{j i}\left(-p_{i j}\right)=b_{i j}-p_{i j}
$$

for all $(i, j) \in E$, where $l_{i j} \leq p_{i j} \leq u_{i j}$ and $a_{i j}, b_{i j} \in \mathbf{R}$, we observe that if $l=u=\mathbf{0}$, we get the marriage model by Gale and Shapley [4]. If $l=(-\mu, \ldots,-\mu)$ and $u=$ $(+\mu, \ldots,+\mu)$ for a sufficiently large $\mu>0$, then we have the assignment model by Shapley and Shubik [10].

We say that a matching $X$ is pairwise-stable if there exists a feasible side payment vector $p$ such that $(X, p)$ is pairwise-stable.

\section{Characterization of Pairwise-stability}

In this section, we characterize pairwise-stability by considering a partition of the set $E$. We will utilize this characterization to develop our algorithm.

Lemma 3.1. A matching $X$ is pairwise-stable if and only if there exist a feasible side payment vector $p$ and two subsets $E_{M}$ and $E_{W}$ of $E$ such that, defining $q$ and $r$ by (2.1) and (2.2), 
(S'1) $q \geq \mathbf{0}$ and $r \geq \mathbf{0}$,

(S'2) $q_{i} \geq \max \left\{\nu_{i j}\left(p_{i j}\right) \mid(i, j) \in E_{M}\right\}$ for all $i \in M$,

(S'3) $r_{j} \geq \max \left\{\nu_{j i}\left(-p_{i j}\right) \mid(i, j) \in E_{W}\right\}$ for all $j \in W$,

(S'4) $E=E_{M} \cup E_{W}$,

(S'5) $p_{i j}=l_{i j}$ for all $(i, j) \in E \backslash E_{M}$ and $p_{i j}=u_{i j}$ for all $(i, j) \in E \backslash E_{W}$,

where we define the maximum over an empty set to be equal to 0.

Proof. $(\Rightarrow)$ Let $(X, \bar{p})$ be a pairwise-stable outcome. We define $q$ and $r$ by $(2.1)$ and (2.2) with $p=\bar{p}$. Let $p$ be a vector defined by

$$
p_{i j}=\left\{\begin{array}{cl}
\nu_{i j}^{-1}\left(q_{i}\right) & \text { if } \nu_{i j}^{-1}\left(q_{i}\right) \in\left[l_{i j}, u_{i j}\right] \\
u_{i j} & \text { if } u_{i j}<\nu_{i j}^{-1}\left(q_{i}\right) \\
l_{i j} & \text { if } \nu_{i j}^{-1}\left(q_{i}\right)<l_{i j}
\end{array} \quad(\forall(i, j) \in E),\right.
$$

and let $E_{M}$ and $E_{W}$ be defined by

$$
\begin{aligned}
& E_{M}=E \backslash\left\{(i, j) \in E \mid \nu_{i j}\left(p_{i j}\right)>q_{i}\right\}, \\
& E_{W}=E \backslash\left\{(i, j) \in E \mid \nu_{j i}\left(-p_{i j}\right)>r_{j}\right\} .
\end{aligned}
$$

Obviously, $p$ is a feasible side payment vector. We will show that $p, E_{M}$ and $E_{W}$ satisfy (S'1) (S'5). Condition (S'1) holds by (S1). Conditions (S'2) and (S'3) are direct consequences of (3.4). We also have (S'4) by (S2) and (3.4). We next show (S'5). Assume $(i, j) \notin E_{M}$, that is, $\nu_{i j}\left(p_{i j}\right)>q_{i}$. By (3.3) and the monotonicity of $\nu_{i j}, \nu_{i j}\left(p_{i j}\right)>q_{i}$ yields $p_{i j}=l_{i j}$. Assume $(i, j) \notin E_{W}$, that is, $\nu_{j i}\left(-p_{i j}\right)>r_{j}$. By (S'4) we see $(i, j) \in E_{M}$, and hence, $\nu_{i j}^{-1}\left(q_{i}\right) \geq l_{i j}$. Suppose $p_{i j}<u_{i j}$ to the contrary. Thus, we have $q_{i}=\nu_{i j}\left(p_{i j}\right)$ by (3.3). Since $r_{j}<\nu_{j i}\left(-p_{i j}\right)$ holds and $\nu_{i j}$ and $\nu_{j i}$ are monotone increasing, there exists a sufficiently small positive number $\varepsilon$ such that $l_{i j} \leq p_{i j}+\varepsilon \leq u_{i j}, q_{i}<\nu_{i j}\left(p_{i j}+\varepsilon\right)$ and $r_{j}<\nu_{j i}\left(-\left(p_{i j}+\varepsilon\right)\right)$. However, this contradicts (S2). Hence $p_{i j}=u_{i j}$ for all $(i, j) \in E \backslash E_{W}$.

$(\Leftarrow)$ We assume that there exist $p, E_{M}$ and $E_{W}$ satisfying (S'1) $\left(\mathrm{S}^{\prime} 5\right)$. We will show that $(X, p)$ is pairwise-stable. Obviously, (S1) holds from (S'1). Suppose to the contrary that (S2) does not hold, i.e., there exist $(i, j) \in E$ and $c \in\left[l_{i j}, u_{i j}\right]$ such that $q_{i}<\nu_{i j}(c)$ and $r_{j}<\nu_{j i}(-c)$. By (S'2), if $q_{i}<\nu_{i j}(c)$ then either (Case 1) $(i, j) \notin E_{M}$ or (Case 2) $(i, j) \in E_{M}$ and $p_{i j}<c$. Similarly by (S'3), if $r_{j}<\nu_{j i}(-c)$ then either (Case 3) $(i, j) \notin E_{W}$ or (Case 4) $(i, j) \in E_{W}$ and $p_{i j}>c$. Trivially (Case 2) and (Case 4) are unsuited. By (S'4), both (Case 1) and (Case 3) do not hold. By (S'5), (Case 1) is irreconcilable to (Case 4), and (Case 2) irreconcilable to (Case 3). This is a contradiction. Hence we have (S2). 


\section{Linear Valuations}

In this section, we deal with the case where for all $(i, j) \in E$, valuations $\nu_{i j}$ and $\nu_{j i}$ are linear, i.e., these are defined by

$$
\nu_{i j}(x)=\alpha_{i j} x+\beta_{i j}, \quad \nu_{j i}(x)=\alpha_{j i} x+\beta_{j i}
$$

where $\alpha_{i j}$ and $\alpha_{j i}$ are given positive reals, and $\beta_{i j}$ and $\beta_{j i}$ are given reals. Our main purpose is to show that a pairwise-stable outcome, namely $\left(X, p, E_{M}, E_{W}\right)$ satisfying $\left(\mathrm{S}^{\prime} 1\right) \sim\left(\mathrm{S}^{\prime} 5\right)$, can be found in polynomial-time in the number of agents. First, we assume that a given $\left(X, p, E_{M}, E_{W}\right)$ satisfies (S'1), (S'3), (S'4), (S'5) and the following condition:

$\left({ }^{\prime} S^{\prime} 2\right) q_{i} \geq \max \left\{\nu_{i j}\left(p_{i j}\right) \mid(i, j) \in E_{M}\right\}$ for each matched man $i$ in $X$.

We note that if no unmatched man in $X$ has any pair in $E_{M} \backslash E_{0}$, then $\left(X, p, E_{M}, E_{W}\right)$ satisfies $\left(\mathrm{S}^{\prime} 1\right) \sim\left(\mathrm{S}^{\prime} 5\right)$, where

$$
E_{0}=\left\{(i, j) \in E \mid \nu_{i j}\left(p_{i j}\right) \leq 0\right\}
$$

One can easily find such a tuple $\left(X, p, E_{M}, E_{W}\right)$ as follows. Define $p \in \mathbf{R}^{E}$ by

$$
p_{i j}:=\left\{\begin{array}{cc}
u_{i j} & \text { if } \nu_{j i}\left(-u_{i j}\right) \geq 0 \\
\max \left\{l_{i j},-\nu_{j i}^{-1}(0)\right\} & \text { if } \nu_{j i}\left(-u_{i j}\right)<0
\end{array}\right.
$$

and define $E_{M}, E_{W}$ by

$$
\begin{aligned}
& E_{M}:=\left\{(i, j) \in E \mid \nu_{j i}\left(-p_{i j}\right) \geq 0\right\} \\
& E_{W}:=\left\{(i, j) \in E \mid p_{i j}<u_{i j}\right\} \cup\left\{(i, j) \in E \mid l_{i j}=u_{i j}, \nu_{j i}\left(-u_{i j}\right)<0\right\} .
\end{aligned}
$$

Obviously, $p$ is a feasible side payment vector. By the definition of $p$, if $\nu_{j i}\left(-p_{i j}\right)<0$ then $p_{i j}=l_{i j}$, and if $p_{i j}<u_{i j}$ then $\nu_{j i}\left(-p_{i j}\right) \leq 0$. These imply that $E_{M}$ and $E_{W}$ satisfy (S'4) and (S'5). To define a matching $X$, we consider $\tilde{q} \in \mathbf{R}^{M}$ and $\widetilde{E}_{M} \subseteq E_{M}$ defined by

$$
\tilde{q}_{i}:=\max \left\{\nu_{i j}\left(p_{i j}\right) \mid(i, j) \in E_{M} \backslash E_{0}\right\}
$$

for all $i \in M$, and

$$
\widetilde{E}_{M}:=\left\{(i, j) \in E_{M} \backslash E_{0} \mid \nu_{i j}\left(p_{i j}\right)=\tilde{q}_{i}\right\} .
$$

Recall that the maximum over an empty set is 0 by definition. We also define a set $\widehat{E}_{M} \subseteq \widetilde{E}_{M}$ as follows:

$$
\widehat{E}_{M}:=\left\{(i, j) \in \widetilde{E}_{M} \mid \nu_{j i}\left(-p_{i j}\right) \geq \nu_{j i^{\prime}}\left(-p_{i^{\prime} j}\right) \forall\left(i^{\prime}, j\right) \in E_{W}\right\} .
$$

By (4.8) and (4.9), $\widehat{E}_{M}$ initially coincides with $\widetilde{E}_{M}$; however, it may be a proper subset of $\widetilde{E}_{M}$ in further iterations in our algorithm. We adopt an artificial notation $\widetilde{W}$ and 
initially put $\widetilde{W}=\emptyset$. Let $X$ be a matching in bipartite graph $\left(M, W ; \widehat{E}_{M}\right)$ such that

$$
\begin{array}{lcl}
X & \text { matches all } & \text { members of } \widetilde{W} \\
X & \text { maximizes } & \sum_{(i, j) \in X} \nu_{j i}\left(-p_{i j}\right) \text { among the matchings having (4.13), } \\
X & \text { maximizes } & \sum_{(i, j) \in X}\left(\ln \alpha_{j i}-\ln \alpha_{i j}\right) \text { among the matchings having (4.14).( }
\end{array}
$$

Since initially $\widetilde{W}=\emptyset$, any matching satisfies (4.13). If there exists a matching satisfying (4.13) then one can easily find a matching $X$ satisfying (4.13), (4.14), and (4.15) by solving the maximum weight matching problem for a bipartite graph. For a matching $X$ defined as above, define $q$ and $r$ by (2.1) and (2.2). Then $q$ is nonnegative because of $\tilde{q} \geq \mathbf{0}$, and $r$ is nonnegative by (4.14), and hence, (S'1) holds. Moreover, (wS'2) holds because of $\tilde{q} \geq \mathbf{0}$, and ( $\left.\mathrm{S}^{\prime} 3\right)$ holds because $\nu_{j i}\left(-p_{i j}\right) \leq 0$ for all $(i, j)$ in $E_{W}$.

Let $\left(X, p, E_{M}, E_{W}\right)$ be a tuple satisfying (S'1), (wS'2), (S'3) (S'5) and, in addition, (4.13) (4.15), where we assume that $\widetilde{W}$ is the set of all matched women in $X$. If (S'2) does not hold, then we modify $\left(X, p, E_{M}, E_{W}\right)$ preserving (S'1), (wS'2), (S'3) (S'5), and $(4.13) \sim(4.15)$. Since we initially put $p$ as large as possible, we monotonically decrease $p$, and hence, preserve $r \geq \mathbf{0}$ and $\nu_{j i}\left(-p_{i j}\right) \geq 0$ for all $(i, j) \in E_{M}$ in our modification. Assume that there exists an unmatched man $i_{0}$ in $X$ such that there is a pair $\left(i_{0}, j\right) \in E_{M} \backslash E_{0}$. Let $D$ be a directed graph $\left(\left\{i_{0}\right\} \cup \widetilde{E}_{M}, A\right)$ with arc set $A$ defined by

$$
\begin{aligned}
A & :=A_{0} \cup A_{1} \cup A_{2} \\
A_{0} & :=\left\{\left(i_{0},\left(i_{0}, j\right)\right) \mid\left(i_{0}, j\right) \in \widetilde{E}_{M}\right\} \\
A_{1} & :=\left\{((i, j),(k, j)) \mid(i, j) \in \widetilde{E}_{M} \backslash X,(k, j) \in X, \nu_{j i}\left(-p_{i j}\right)=\nu_{j k}\left(-p_{k j}\right)\right\} \\
A_{2} & :=\left\{((i, j),(i, k)) \mid(i, j) \in X,(i, k) \in \widetilde{E}_{M} \backslash X\right\}
\end{aligned}
$$

and assign weights $w(e)$ to each $\operatorname{arc} e$ of $D$ as follows:

$$
\begin{aligned}
e=\left(i_{0},\left(i_{0}, j\right)\right) \in A_{0} & \Rightarrow w(e)=\ln \alpha_{i_{0} j}, \\
e=((i, j),(k, j)) \in A_{1} & \Rightarrow w(e)=-\ln \alpha_{j i}+\ln \alpha_{j k}, \\
e=((i, j),(i, k)) \in A_{2} & \Rightarrow w(e)=-\ln \alpha_{i j}+\ln \alpha_{i k} .
\end{aligned}
$$

Let

$$
R\left(i_{0}\right)=\left\{(i, j) \in \widetilde{E}_{M} \mid(i, j) \text { is reachable from } i_{0} \text { in } D\right\}
$$

By the definition of $D$, we have the following lemma.

Lemma 4.1. Assume that $(i, j) \in R\left(i_{0}\right) \backslash X$ and $(k, j) \in X$. Then, we have $\nu_{j i}\left(-p_{i j}\right) \leq$ $\nu_{j k}\left(-p_{k j}\right)$. 
Proof. Suppose to the contrary that $(i, j) \in R\left(i_{0}\right) \backslash X$ and $\nu_{j i}\left(-p_{i j}\right)>\nu_{j k}\left(-p_{k j}\right)$ for $(k, j) \in X$. Since $(i, j)$ is reachable from $i_{0}$, there exists a sequence $S$ of pairs in $\widetilde{E}_{M}$ :

$$
S=\left(i_{0}, j_{0}\right),\left(i_{1}, j_{0}\right),\left(i_{1}, j_{1}\right), \ldots,\left(i_{s}, j_{s}\right)=(i, j),\left(i_{s+1}, j_{s}\right)=(k, j)
$$

such that $\left(\left(i_{h}, j_{h}\right),\left(i_{h+1}, j_{h}\right)\right) \in A_{1}$ for $h=0,1, \ldots, s-1$ and $\left(\left(i_{h}, j_{h-1}\right),\left(i_{h}, j_{h}\right)\right) \in A_{2}$ for $h=1, \ldots, s$. Obviously, the symmetric difference $X^{\prime}$ of $S$ and $X$ is a matching covering $\widetilde{W}$, and $X^{\prime}$ is strictly greater than $X$ in the sense of (4.14). This, however, is a contradiction. Thus, the assertion holds.

We also have the following lemma.

Lemma 4.2. $D$ has no negative cycle with respect to $w$.

Proof. Assume that $D$ has a negative cycle $C$. By the definition of $D$, vertices corresponding to $\widetilde{E}_{M} \backslash X$ and $X$ alternately appear in $C$. We express $C$ by a sequence of pairs of $\widetilde{E}_{M}$ as

$$
C=\left(i_{1}, j_{1}\right),\left(i_{2}, j_{1}\right),\left(i_{2}, j_{2}\right), \ldots,\left(i_{s}, j_{s}\right),\left(i_{s+1}, j_{s}\right)=\left(i_{1}, j_{s}\right),\left(i_{s+1}, j_{s+1}\right)=\left(i_{1}, j_{1}\right),
$$

where $\left(i_{h}, j_{h}\right) \in \widetilde{E}_{M} \backslash X$ and $\left(i_{h+1}, j_{h}\right) \in X$ for all $h=1,2, \ldots, s$. By (4.17), the weight $w(C)$ of $C$ is calculated as

$$
\begin{aligned}
w(C) & =\sum_{h=1}^{s}\left(-\ln \alpha_{j_{h} i_{h}}+\ln \alpha_{j_{h} i_{h+1}}-\ln \alpha_{i_{h+1} j_{h}}+\ln \alpha_{i_{h+1} j_{h+1}}\right) \\
& =\sum_{(i, j) \in C \cap X}\left(\ln \alpha_{j i}-\ln \alpha_{i j}\right)-\sum_{(i, j) \in C \backslash X}\left(\ln \alpha_{j i}-\ln \alpha_{i j}\right)<0 .
\end{aligned}
$$

By the construction of $D$, the symmetric difference $X^{\prime}$ of $X$ and $C$, which is a matching in $\left(M, W ; \widehat{E}_{M}\right)$, also satisfies (4.13) and (4.14). The assumption that $w(C)<0$, however, implies that

$$
\sum_{(i, j) \in X}\left(\ln \alpha_{j i}-\ln \alpha_{i j}\right)<\sum_{(i, j) \in X^{\prime}}\left(\ln \alpha_{j i}-\ln \alpha_{i j}\right)
$$

which contradicts (4.15). Hence, $D$ has no negative cycle.

By Lemma 4.2, we can consider the shortest distance $d: \widetilde{E}_{M} \rightarrow \mathbf{R} \cup\{+\infty\}$ from $i_{0}$ to the other vertices in $D$ with respect to $w$. For convenience, we denote the shortest distance of $(i, j) \in \widetilde{E}_{M}$ by $d_{(i, j)}$. We now decrease $p$ with a parameter $\varepsilon \geq 0$ as

$$
p_{i j}(\varepsilon):= \begin{cases}p_{i j}-\varepsilon \exp \left(-d_{(i, j)}\right) & \text { if }(i, j) \in R\left(i_{0}\right) \\ p_{i j} & \text { otherwise. }\end{cases}
$$

Before discussing how to determine a parameter $\varepsilon$, we give two lemmas.

Lemma 4.3. For any $(i, j) \in R\left(i_{0}\right)$, we have $(i, k) \in R\left(i_{0}\right)$ for all $(i, k) \in \widetilde{E}_{M}$ and $\nu_{i j}\left(p_{i j}(\varepsilon)\right)=\nu_{i k}\left(p_{i k}(\varepsilon)\right)$, where $\varepsilon \geq 0$. 
Proof. If $(i, j) \in X$ then $((i, j),(i, k)) \in A_{2}$, and hence $(i, k) \in R\left(i_{0}\right)$. We assume that $(i, j) \in R\left(i_{0}\right) \backslash X$. If $i=i_{0}$ then $(i,(i, k)) \in A_{0}$ and hence $(i, k) \in R\left(i_{0}\right)$. Assume that $i \neq i_{0}$. By the construction of $D$, given a vertex of $\widetilde{E}_{M} \backslash X$ in $D$, if it has an entering arc, then the arc is unique and leaves some vertex of $X$. Since $(i, j) \in R\left(i_{0}\right)$, there exists $\left(i, j^{\prime}\right) \in X \cap R\left(i_{0}\right)$ such that $\left(\left(i, j^{\prime}\right),(i, j)\right) \in A_{2}$, and hence $(i, k) \in R\left(i_{0}\right)$ for all $(i, k) \in \widetilde{E}_{M}$.

We next show that $\nu_{i j}\left(p_{i j}(\varepsilon)\right)=\nu_{i k}\left(p_{i k}(\varepsilon)\right)$. If $i=i_{0}$ then the unique path from $i_{0}$ to $\left(i_{0}, j\right)$ is $\left(i_{0},\left(i_{0}, j\right)\right)$ and hence

$$
\nu_{i_{0} j^{\prime}}\left(p_{i_{0} j^{\prime}}(\varepsilon)\right)=\alpha_{i_{0} j^{\prime}}\left(p_{i_{0} j^{\prime}}-\varepsilon \exp \left(-\ln \alpha_{i_{0} j^{\prime}}\right)\right)+\beta_{i_{0} j^{\prime}}=\nu_{i_{0 j^{\prime}}}\left(p_{i_{0} j^{\prime}}\right)-\varepsilon
$$

for all $\left(i_{0}, j^{\prime}\right) \in \widetilde{E}_{M}$, which means $\nu_{i_{0} j}\left(p_{i_{0} j}(\varepsilon)\right)=\nu_{i_{0} k}\left(p_{i_{0} k}(\varepsilon)\right)$. Assume that $i \neq i_{0}$, and, without loss of generality, assume that $(i, j) \in X$. For each $(i, k) \in \widetilde{E}_{M} \backslash X$, we have $d_{(i, k)}=d_{(i, j)}+\left(-\ln \alpha_{i j}+\ln \alpha_{i k}\right)$. Hence, we have

$$
\begin{aligned}
\nu_{i k}\left(p_{i k}(\varepsilon)\right) & =\alpha_{i k}\left(p_{i k}-\varepsilon \exp \left(-d_{(i, j)}+\ln \alpha_{i j}-\ln \alpha_{i k}\right)\right)+\beta_{i k} \\
& =\nu_{i k}\left(p_{i k}\right)-\varepsilon \alpha_{i j} \exp \left(-d_{(i, j)}\right)=\nu_{i j}\left(p_{i j}(\varepsilon)\right) .
\end{aligned}
$$

This completes the proof.

Lemma 4.4. Assume that $(i, j) \in R\left(i_{0}\right)$, and that there exists $(k, j) \in X$. For a sufficiently small $\varepsilon \geq 0, \nu_{j i}\left(-p_{i j}(\varepsilon)\right) \leq \nu_{j k}\left(-p_{k j}(\varepsilon)\right)$ holds. Moreover, if $(k, j) \in X \cap$ $R\left(i_{0}\right)$, then the above inequality holds for all $\varepsilon \geq 0$. The above inequality holds with equality if arc $((i, j),(k, j))$ lies on a shortest path from $i_{0}$ to $(k, j)$.

Proof. In the case where $\nu_{j i}\left(-p_{i j}\right)<\nu_{j k}\left(-p_{k j}\right)$, the assertion obviously holds. In the other case, it follows from Lemma 4.1 that $\nu_{j i}\left(-p_{i j}\right)=\nu_{j k}\left(-p_{k j}\right)$, i.e., $(k, j) \in X \cap R\left(i_{0}\right)$. Since $d$ is the shortest distance with respect to $w$, we have $d_{(k, j)} \leq d_{(i, j)}-\ln \alpha_{j i}+\ln \alpha_{j k}$. Hence, we have

$$
\begin{aligned}
\nu_{j k}\left(-p_{k j}(\varepsilon)\right) & =\alpha_{j k}\left(-p_{k j}+\varepsilon \exp \left(-d_{(k, j)}\right)\right)+\beta_{j k} \\
& =\nu_{j i}\left(-p_{i j}\right)+\varepsilon \alpha_{j k} \exp \left(-d_{(k, j)}\right) \\
& \geq \nu_{j i}\left(-p_{i j}\right)+\varepsilon \alpha_{j k} \exp \left(-d_{(i, j)}+\ln \alpha_{j i}-\ln \alpha_{j k}\right)=\nu_{j i}\left(-p_{i j}(\varepsilon)\right) .
\end{aligned}
$$

Note that if $((i, j),(k, j))$ lies on a shortest path from $i_{0}$ to $(k, j)$, then $d_{(k, j)}=d_{(i, j)}-$ $\ln \alpha_{j i}+\ln \alpha_{j k}$ and hence $\nu_{j k}\left(-p_{k j}(\varepsilon)\right)=\nu_{j i}\left(-p_{i j}(\varepsilon)\right)$. This completes the proof.

Let

$$
\widetilde{W}:=\{j \in W \mid j \text { is matched in } X\}
$$

be the set of all matched women for the matching $X$. Our aim is to decrease $p$ as (4.19) by a parameter $\varepsilon \geq 0$. Note that $\nu_{i j}$ and $\nu_{j i}$, for all $(i, j) \in E$ are monotone increasing on $\mathbf{R}$ and $\nu_{i j}\left(p_{i j}\right)>0$ for all $(i, j) \in E \backslash E_{0}$. For the time being, if we ignore the lower bound $l$ of the side payment vector, then we have the following bounds on the values of $\nu_{i j}\left(p_{i j}\right)$ and $\nu_{j i}\left(-p_{i j}\right)$ for any $(i, j) \in R\left(i_{0}\right)$ : 
(a) There exists $\tilde{p}_{i j} \in\left(-\infty, p_{i j}\right]$ such that $\nu_{i j}\left(\tilde{p}_{i j}\right)=0$.

(b) If there exists $(i, k) \in E_{M} \backslash E_{0}$ such that $(i, k) \notin \widetilde{E}_{M}$ or $k \notin \widetilde{W}$, then there exists $\tilde{p}_{i j} \in\left(-\infty, p_{i j}\right]$ such that $\nu_{i j}\left(\tilde{p}_{i j}\right)=\nu_{i k}\left(p_{i k}\right)$.

(c) If $(i, j) \in R\left(i_{0}\right) \backslash X$, then for $(k, j) \in X \backslash R\left(i_{0}\right)$, there exists $\tilde{p}_{i j} \in\left(-\infty, p_{i j}\right]$ such that $\nu_{j i}\left(-\tilde{p}_{i j}\right)=\nu_{j k}\left(-p_{k j}\right)$. Note that Lemma 4.1 guarantees the existence of such $\tilde{p}_{i j}$ provided that $(k, j)$ exists.

To decide parameter $\varepsilon$, we explicitly write the above here:

(Case 1) Let $\varepsilon_{1} \geq 0$ be the minimum for which $\nu_{i j}\left(p_{i j}\left(\varepsilon_{1}\right)\right)=\nu_{i k}\left(p_{i k}\left(\varepsilon_{1}\right)\right)$ for some $(i, j) \in R\left(i_{0}\right)$ and $(i, k) \in E_{M}$ with $k \in W \backslash \widetilde{W}$. If such $\varepsilon_{1}$ does not exist, then put $\varepsilon_{1}=+\infty$.

(Case 2) Let $\varepsilon_{2} \geq 0$ be the minimum for which $\nu_{i j}\left(p_{i j}\left(\varepsilon_{2}\right)\right)=\nu_{i k}\left(p_{i k}\left(\varepsilon_{2}\right)\right)$ for some $(i, j) \in R\left(i_{0}\right)$ and $(i, k) \in E_{M} \backslash R\left(i_{0}\right)$ with $k \in \widetilde{W}$. If such $\varepsilon_{2}$ does not exist, then put $\varepsilon_{2}=+\infty$.

(Case 3) Let $\varepsilon_{3} \geq 0$ be the minimum for which $\nu_{i j}\left(p_{i j}\left(\varepsilon_{3}\right)\right)=0$ for some $(i, j) \in R\left(i_{0}\right)$.

(Case 4) Let $\varepsilon_{4} \geq 0$ be the minimum for which $p_{i j}\left(\varepsilon_{4}\right)=l_{i j}$ holds for some $(i, j) \in$ $R\left(i_{0}\right)$.

(Case 5) Let $\varepsilon_{5} \geq 0$ be the minimum for which $\nu_{j i}\left(-p_{i j}\left(\varepsilon_{5}\right)\right)=\nu_{j k}\left(-p_{k j}\left(\varepsilon_{5}\right)\right)$ holds for some $(i, j) \in R\left(i_{0}\right) \backslash X$ and $(k, j) \in X \backslash R\left(i_{0}\right)$. If such $\varepsilon_{5}$ does not exist, then put $\varepsilon_{5}=+\infty$.

Since $l \in \mathbf{R}^{E}$, we have $\varepsilon_{4} \in \mathbf{R}$. Thus, $\varepsilon$ determined by

$$
\varepsilon=\min \left\{\varepsilon_{1}, \varepsilon_{2}, \varepsilon_{3}, \varepsilon_{4}, \varepsilon_{5}\right\}
$$

is well-defined. It follows from $\varepsilon \leq \varepsilon_{4}$ that $p(\varepsilon)$ is a feasible side payment vector. Put

$$
\tilde{p}=p(\varepsilon) .
$$

Now, our algorithm is described as below.

\section{Algorithm Stable_Outcome}

Step 0: Let $\widetilde{W}=\emptyset$. Initially define $\left(X, p, E_{M}, E_{W}\right), E_{0}$ and $\widetilde{E}_{M}$ by (4.6), (4.7), (4.8), (4.9), (4.11), and (4.13) (4.15).

Step 1: If no unmatched man in $X$ has any pair in $E_{M} \backslash E_{0}$ then stop.

Step 2: Let $i_{0}$ be an unmatched man in $X$ such that there is a pair $\left(i_{0}, j\right) \in E_{M} \backslash E_{0}$. Construct a directed graph $D=\left(\left\{i_{0}\right\} \cup \widetilde{E}_{M}, A\right)$ and weight $w$ by (4.11), (4.16) and (4.17). Calculate the shortest distances $d$ of all vertices in $D$ from $i_{0}$, and $\varepsilon$ and $\tilde{p}$ by (4.22) and (4.23). 
Step 3: Let $R\left(i_{0}\right)$ and $\widetilde{W}$ be the sets defined by (4.18) and (4.21) for the current matching $X$. Suppose that $(i, j)$ and $k$ denote a pair and an agent defined in the above five cases.

(a) If $\varepsilon=\varepsilon_{1}$ then set $\widetilde{W}:=\widetilde{W} \cup\{k\}$ and go to Step 4; else go to (b),

(b) If $\varepsilon=\varepsilon_{2}$ then go to Step 4; else go to (c),

(c) If $\varepsilon=\varepsilon_{3}$ then set $E_{0}:=E_{0} \cup\left\{\left(i, j^{\prime}\right) \in \widetilde{E}_{M} \mid j^{\prime} \in W\right\}$ and go to Step 4; else go to $(d)$,

(d) If $\varepsilon=\varepsilon_{4}$ then set $E_{M}:=E_{M} \backslash\{(i, j)\}$ and $E_{W}:=E_{W} \cup\{(i, j)\}$ and go to Step 4; else go to (e),

(e) If $\varepsilon=\varepsilon_{5}$ then go to Step 4,

Step 4: Replace $p$ by $\tilde{p}$, and calculate $\widetilde{E}_{M}$ and $\widehat{E}_{M}$ by (4.11) and (4.12) for the updated $p$. Redefine $E_{W}$ by (4.9). Find a matching $X$ in $\left(M, W ; \widehat{E}_{M}\right)$ satisfying $(4.13) \sim(4.15)$. Go to Step 1 .

We first show that Stable_Outcome works correctly.

Lemma 4.5. In each iteration of Stable_Outcome, $\left(X, p, E_{M}, E_{W}\right)$ at Step 1 satisfies (S'1), (wS'2), (S'3) (S'5) and (4.13) (4.15), and furthermore, there exists a matching in $\left(M, W ; \widehat{E}_{M}\right)$ satisfying $(4.13) \sim(4.15)$ at Step 4.

Proof. By the initial selection of $\left(X, p, E_{M}, E_{W}\right)$ at Step 0, obviously (S'1), (wS'2) and $\left(\mathrm{S}^{\prime} 3\right) \sim\left(\mathrm{S}^{\prime} 5\right)$ and $(4.13) \sim(4.15)$ hold prior to the execution of Step 1 in the first iteration. Thus, the first assertion holds in the first iteration.

Suppose that $\left(X, p, E_{M}, E_{W}\right)$ satisfies (S'1), (wS'2) and (S'3) (S'5) and (4.13) (4.15) before the start of the th-iteration, $t \geq 1$. We first show the second assertion. It is enough to show that there exists a matching $\widetilde{X}$ in $\left(M, W ; \widehat{E}_{M}\right)$ satisfying (4.13). We consider Cases 2 and 5. In these cases, by Lemma 4.3, $\widetilde{E}_{M}$ remains the same or enlarges at Step 4. Furthermore, Lemma 4.4 and (S'3) guarantee $X \subseteq \widehat{E}_{M}$ for the updated $\widehat{E}_{M}$ because the new pairs added into $E_{W}$ belong to $R\left(i_{0}\right)$. Hence $X$ satisfies (4.13) in Cases 2 and 5. Analogously, $X$ satisfies (4.13) when $(i, j) \notin X$ in Case 4 . We next consider Cases 3 and 4 with $(i, j) \in X$. In these cases, there exists a shortest path $S$ from $i_{0}$ to $(i, j)$ in $D$, which is denoted by

$$
S=\left(i_{0}, j_{0}\right),\left(i_{1}, j_{0}\right),\left(i_{1}, j_{1}\right), \ldots,\left(i_{s}, j_{s}\right)\left(i_{s+1}, j_{s}\right)=(i, j)
$$

where $\left(\left(i_{h}, j_{h}\right),\left(i_{h+1}, j_{h}\right)\right) \in A_{1}$ for $h=0, \ldots, s$ and $\left(\left(i_{h}, j_{h-1}\right),\left(i_{h}, j_{h}\right)\right) \in A_{2}$ for $h=$ $1, \ldots, s$. In the same way as above, Lemmas $4.3,4.4$ and (S'3) guarantee that all pairs in $S \cup X$ other than $(i, j)$ are contained in $\widehat{E}$. Let $\widetilde{X}$ be the symmetric difference of $S$ and $X$. Obviously, $(i, j) \notin \widetilde{X}, \widetilde{X} \cap E_{0}=\emptyset$, and $\widetilde{X}$ is a matching in $\left(M, W ; \widehat{E}_{M}\right)$ satisfying (4.13). We finally consider Case 1 . In this case, $(i, k)$ belongs to the updated $\widehat{E}$ because of $\nu_{k i}\left(-p_{i k}\right) \geq 0$ and (S'3). By the assumption of Case 1 , there exists a pair 
$(i, j) \in X$ which is reachable from $i_{0}$. Let $S$ be a shortest path from $i_{0}$ to $(i, j)$ denoted as (4.24). We can show that $S \cup X$ belongs to $\widehat{E}_{M}$ as above. Thus, the symmetric difference $\widetilde{X}$ of $S \cup\{(i . j)\}$ and $X$ is a matching covering the updated $\widetilde{W}$.

Next we prove that $\left(X, p, E_{M}, E_{W}\right)$ defined at Step 4 satisfies (S'1), (wS'2) and (S'3) $\sim$ (S'5). We have $q \geq \mathbf{0}$ since $\varepsilon \leq \varepsilon_{3}$, and $r \geq \mathbf{0}$ since (4.14) holds for $X$ and $\nu_{j i}\left(-p_{i j}\right) \geq 0$ for all $(i, j) \in E_{M}$. Hence (S'1) does hold. Definitions of $\tilde{q}$ and $\widehat{E}_{M}$, and the fact that $X \subseteq \widehat{E}_{M}$ yield (wS'2) and (S'3). At Step 3, $E_{M}$ and $E_{W}$ remain the same or one element is removed from $E_{M}$ and added into $E_{W}$, and at Step $4 E_{W}$ may be enlarged. Thus (S'4) holds true. We note that if any $(i, j) \in E$ is removed from $E_{M}$ and added into $E_{W}$, then $p_{i j}=l_{i j}$. Hence, the first part of (S'5) holds. Also $(i, j) \in E_{W}$ if and only if either $p_{i j}<u_{i j}$ or $\left(l_{i j}=u_{i j}\right.$ and $\left.\nu_{j i}\left(-u_{i j}\right)<0\right)$. Thus, we have $p_{i j}=u_{i j}$ for all $(i, j) \in E \backslash E_{W}$, the second part of (S'5). This completes the proof.

Theorem 4.6. If StABle_Outcome terminates, then it outputs a pairwise-stable outcome $\left(X, p, E_{M}, E_{W}\right)$ satisfying (S'1) (S'5).

Proof. Lemma 4.5 guarantees that $\left(X, p, E_{M}, E_{W}\right)$ at Step 1 satisfies (S'1), (wS'2) and (S'3) (S'5). If Stable_Outcome terminates, then there is no unmatched man in $X$ having any pair in $E_{M} \backslash E_{0}$. This says that $\left(X, p, E_{M}, E_{W}\right)$ also satisfies (S'2).

Until now, we have spent our efforts to show the correctness of StABle_Outcome. We now finally show that Stable_Outcome terminates in polynomial-time in the number $n$ of agents.

Lemma 4.7. Stable_Outcome terminates in $O\left(n^{3}\right)$ iterations.

Proof. During Stable_Outcome, $\widetilde{W}, E_{0}$ and $E_{W}$ enlarge or remain the same, and a pair eliminated from $\widetilde{E}_{M}$ will never appear again in $\widetilde{E}_{M}$.

If $\varepsilon=\varepsilon_{1}$ then $\widetilde{W}$ is enlarged. Thus, Case 1 occurs at most $|W|$ times.

If $\varepsilon=\varepsilon_{2}$, then at least one pair is added to $\widetilde{E}_{M}$, and hence, Case 2 occurs at most $|E|$ times.

Since $E_{0}$ is enlarged if $\varepsilon=\varepsilon_{3}$, Case 3 occurs at most $|M|$ times.

If $\varepsilon=\varepsilon_{4}$, then one pair is deleted from $E_{M}$ and added in $E_{W}$ and is not selected for $X$ in the subsequent iterations. Case 4 occurs at most $|E|$ times.

Summing up the above discussion, the cases other than Case 5 occurs at most $O\left(n^{2}\right)$ times.

We finally consider the case where Case 5 occurs but the other cases do not occur. In this case, a pair in $X$ is added into $R\left(i_{0}\right)$. Such case successively occurs at most $|\widetilde{W}|$ times, because $R\left(i_{0}\right)$ does not reduce if Cases $1,2,3$, and 4 do not occur. This means that Stable_Outcome terminates in $O\left(n^{3}\right)$ iterations.

In each iteration of Stable_Outcome, we solve the maximum weight matching problem in bipartite graph $\left(M, W ; \widehat{E}_{M}\right)$ and the single source shortest path problem in $D$. We note that one can execute Stable_Outcome without calculating logarithms 
nor exponentials in practice, because maximizing $\sum_{(i, j) \in X}\left(\ln \alpha_{j i}-\ln \alpha_{i j}\right)$ is equivalent to maximizing $\Pi_{(i, j) \in X} \frac{\alpha_{j i}}{\alpha_{i j}}$, and because $\exp \left(-d_{(i, j)}\right)$ in (4.19) can be expressed by products and divisions of $\alpha_{i j}$ 's. It is well known that the maximum weight matching problem in a bipartite graph can be solved in $O\left(n^{3}\right)$. Since the arcs in graph $D$ have general weights (positive or negative) and $D$ does not have negative cycles with respect to $w$, we utilize the Moore-Bellman-Ford Algorithm which finds the shortest distances in $O\left(\left|\widetilde{E}_{M}\right| \cdot|A|\right)$. Since each pair $(i, j) \in \widetilde{E}_{M} \backslash X$ has at most one entering arc and one leaving arc in $D,|A|$ is bounded by $n^{2}$ from above. Thus, the shortest distances can be calculated in $O\left(n^{4}\right)$. By Lemma 4.7, we can easily derive the following Theorem.

Theorem 4.8. The complexity of Stable_Outcome is $O\left(n^{7}\right)$ where $n$ denotes the number of agents.

\section{Acknowledgements}

The author is grateful to Satoru Fujishige and Akihisa Tamura for their skilful guidance which paved the way for this research task.

\section{References}

[1] K. Eriksson, and J. Karlander, Stable matching in a common generalization of the marriage and assignment models, Discrete Math. 217 (2000), 135-156.

[2] S. Fujishige and A. Tamura, A general two-sided matching market with discrete concave utility functions, Discrete Appl. Math. 154 (2006), 950-970.

[3] S. Fujishige and A. Tamura, A two-Sided discrete-concave market with bounded side payments: An approach by discrete concave analysis, Math. Oper. Res. (to appear).

[4] D. Gale and L. S. Shapley, College admissions and the stability of marriage, Amer. Math. Monthly 69 (1962), 9-15.

[5] M. Kaneko, The central assignment game and the assignment markets, J. Math. Econom. 10 (1982), 205-232.

[6] K. Murota, Convexity and Steinitz's exchange property, Adv. Math. 124 (1996), $272-311$.

[7] K. Murota, Discrete convex analysis, Math. Programming 83 (1998), 313-371.

[8] K. Murota, Discrete Convex Analysis, Society for Industrial and Applied Mathematics, Philadelphia, 2003.

[9] A. E. Roth and M. A. O. Sotomayor, Stable outcomes in discrete and continuous models of two-sided matching: A unified treatment, Rev. Econom. 16 (1996), 1-24. 
[10] L. S. Shapley and M. Shubik, The assignment game I: The core, Internat. J. Game Theory 1 (1972), 111-130.

[11] M. Sotomayor, Existence of stable outcomes and the lattice property for a unified matching market, Math. Social Sci. 39 (2000), 119-132. 